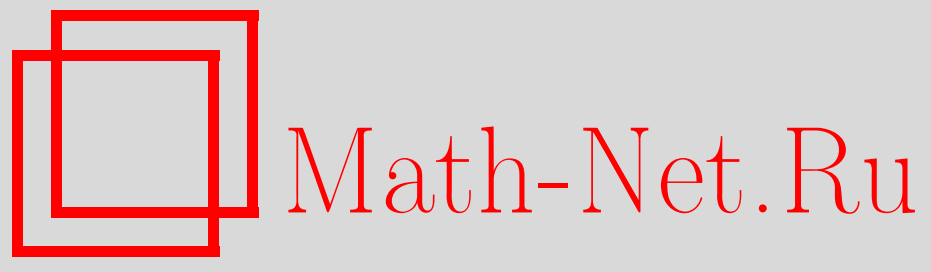

А. М. Цейтлин, Квантование $N=2$ суперсимметричной иерархии КдФ, ТМФ, 2006, том 147, номер 2, 303-314 DOI: https://doi.org/10.4213/tmf1965

Использование Общероссийского математического портала Math-Net.Ru подразумевает, что вы прочитали и согласны с пользовательским соглашением http: //www . mathnet.ru/rus/agreement

Параметры загрузки:

IP: 18.234 .156 .22

26 апреля 2023 г., 13:41:40

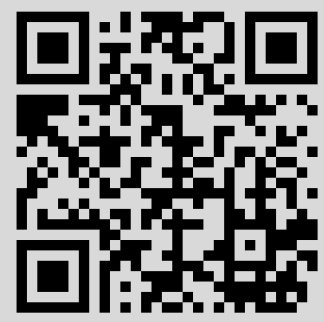




\title{
КВАНТОВАНИЕ $N=2$ СУПЕРСИММЕТРИЧНОЙ ИЕРАРХИИ КДФ
}

\begin{abstract}
Продолжено изучение процедуры квантования суперсимметричных интегрируемых иерархий КдФ. Рассмотрена $N=2$ суперсимметричная система КдФ, базирующаяся на аффинной супералгебре $s l^{(1)}(2 \mid 1)$ с новой алгебраической конструкцией $L$-оператора, отличной от стандартной редукции ДринфельдаСоколова. Построена квантовая матрица монодромии, удовлетворяющая специальной версии уравнения отражения и показано, что в классическом пределе этот объект дает в точности матрицу монодромии $N=2$ суперсимметричной системы КдФ. Показано, что как на квантовом, так и на классическом уровне след матрицы монодромии (трансфер-матрица) инвариантен относительно двух суперпреобразований и нулевой моды соответствующего $U(1)$-тока.
\end{abstract}

Ключевые слова: суперконформная теория поля, квантовые супералгебры, суперсимметричное уравнение КдФ, интегрируемые суперсимметричные системы, квантование.

\section{1. ВВЕДЕНИЕ}

В предыдущих работах [1]-[3] мы рассматривали квантование иерархий Дринфельда-Соколова, связанных с аффинными алгебрами, в соответствии с подходом, предложенным в [4] для обычной $\widehat{s l}(2)$-модели КдФ. В этой статье мы расширяем этот подход для нестандартных иерархий КдФ, связанных с аффинными супералгебрами $s l^{(1)}(m+1 \mid m)$. Мы подробно рассматриваем $N=2$ суперсимметричную модель КдФ ( $N=2$ СУСИ-КдФ-модель), построенную с помощью супералгебры $s l^{(1)}(2 \mid 1)$, при этом все конструкции прямо обобщаются на случай старшего ранга. Интерес к таким интегрируемым моделям вызван тем, что процедура квантования становится более содержательной по сравнению со стандартными иерархиями Дринфельда-Соколова.

Для того чтобы приступить к квантованию данной модели, мы начинаем с соответствующей классической теории (см. раздел 2). Классическая версия ассоциированной матрицы монодромии представлена посредством известной Р-экспоненты, но ее показатель состоит не только из генераторов, соответствующих простым корням и их квадратичным комбинациям, но также из генераторов, соответствующих

* Санкт-Петербургское отделение математического института им. В. А. Стеклова РАН, Санкт-Петербург, Россия. E-mail: zam@math.ipme.ru 
более сложным составным корням. Мы доказываем, что, как в "стандартном" случае, все члены, соответствующие составным корням, исчезают из первой итерации квантового обобщения Р-экспоненты; кроме того, мы показываем, что эта квантовая Р-экспонента совпадает с редуцированной универсальной $R$-матрицей, связанной с квантовой супералгеброй $\widehat{s l}_{q}(2 \mid 1)$ (см. разделы 3,4$)$. Мы также доказываем, что суперследы квантовой версии матрицы монодромии, так называемые трансферматрицы (по очевидной аналогии с решеточным случаем), коммутируют с генераторами суперсимметрии. Следовательно, эти генераторы могут быть включены в семейство интегралов движения как на классическом, так и на квантовом уровне (см. раздел 5). В разделе 5 мы обсуждаем связь $N=2$ СУСИ-КдФ-модели с топологическими теориями и их интегрируемыми возмущениями.

\section{2. НЕСТАНДАРТНАЯ ФОРМА ИЕРАРХИИ $N=2$ СУСИ-КДФ-МОДЕЛИ}

Матричный $L$-оператор для $N=2$ СУСИ-КдФ-модели имеет следующий явный вид [5]:

$$
\mathcal{L}_{F}=D-\left(\begin{array}{ccc}
D \Phi_{1} & 1 & 0 \\
\lambda & D \Phi_{1}-D \Phi_{2} & 1 \\
\lambda D\left(\Phi_{1}-\Phi_{2}\right) & \lambda & -D \Phi_{2}
\end{array}\right)
$$

где $D=\partial_{\theta}+\theta \partial_{u}, u$ - переменная на цилиндре с окружностью $2 \pi, \theta$ - грассманово число, а $\Phi_{j}(u, \theta)=\phi_{j}(u)-(i / \sqrt{2}) \theta \xi_{j}(u), j=1,2$, являются суперполями со следующими скобками Пуассона:

$$
\begin{aligned}
& \left\{D_{u_{1}, \theta_{1}} \Phi_{j}\left(u_{1}, \theta_{1}\right), D_{u_{2}, \theta_{2}} \Phi_{j}\left(u_{2}, \theta_{2}\right)\right\}=0, \quad j=1,2, \\
& \left\{D_{u_{1}, \theta_{1}} \Phi_{1}\left(u_{1}, \theta_{1}\right), D_{u_{2}, \theta_{2}} \Phi_{2}\left(u_{2}, \theta_{2}\right)\right\}=-D_{u_{1}, \theta_{1}}\left(\delta\left(u_{1}-u_{2}\right)\left(\theta_{1}-\theta_{2}\right)\right) .
\end{aligned}
$$

Оператор (1) есть нечетный $L$-оператор, связанный с аффинной супералгеброй $s l^{(1)}(2 \mid 1)$ (элементы в столбце вектора представления градуированы сверху вниз в следующем порядке: четный, нечетный, четный). Существует еще один (канонический) $L$-оператор, также соответствующий $N=2$ СУСИ-КдФ-модели, связанный с супералгеброй $s l^{(1)}(2 \mid 2)[6]$. Квантование этой реализации, соответствующей более высокому рангу, может быть осуществлено посредством процедуры, намеченной в [2], [3], и ничего нового по сравнению с [1]-[3] не добавляет, более того, теория представлений соответствующей супералгебры $s l^{(1)}(2 \mid 2)$ достаточно сложна как в классическом, так и в квантовом случае.

Предъявленная форма $L$-оператора, соответствующая более низкому рангу, позволяет работать с более простой теорией представлений супералгебры $s l^{(1)}(2 \mid 1)$ и, кроме того, дает возможность увидеть новые интересные аспекты формализма квантования.

Для построения скалярного $L$-оператора, связанного с матричным, рассмотрим линейную задачу $\mathcal{L} \Psi=0$ и выразим второй и третий элементы в векторе $\Psi$ в терминах первого (верхнего). Линейное уравнение для этого элемента имеет следующий вид:

$$
\left(\left(D+D \Phi_{1}\right)\left(D-D\left(\Phi_{1}-\Phi_{2}\right)\right)\left(D-D \Phi_{2}\right)+2 \lambda D\right) \Psi_{1}=0
$$


Таким образом, мы получаем скалярный $L$-оператор

$$
L=D^{3}+(\mathcal{V}+2 \lambda) D+\mathcal{U},
$$

где преобразование Миуры задано следующими соотношениями:

$$
\begin{gathered}
\mathcal{V}=-D \Phi_{2} D \Phi_{1}+\partial \Phi_{2}-\partial \Phi_{1}=\frac{i}{\sqrt{2}} \theta\left(\alpha^{+}-\alpha^{-}\right)+V, \\
\mathcal{U}=-\partial \Phi_{2} D \Phi_{1}-D \partial \Phi_{1}=\theta U+\frac{i}{\sqrt{2}} \alpha^{+}, \\
U=-\phi_{1}^{\prime} \phi_{2}^{\prime}-\frac{1}{2} \xi_{1} \xi_{2}^{\prime}-\phi_{1}^{\prime \prime}, \quad V=\phi_{2}^{\prime}-\phi_{1}^{\prime}+\frac{1}{2} \xi_{2} \xi_{1}, \\
\alpha^{+}=\xi_{1} \phi_{2}^{\prime}+\xi_{1}^{\prime}, \quad \alpha^{-}=\xi_{2} \phi_{1}^{\prime}+\xi_{2}^{\prime},
\end{gathered}
$$

причем $U, V, \alpha^{ \pm}$удовлетворяют $N=2$ суперконформной алгебре со скобками Пуассона

$$
\begin{aligned}
\left\{U(u), \alpha^{+}(v)\right\} & =-\alpha^{+}(u) \delta(u-v)-2 \alpha^{+}(u) \delta^{\prime}(u-v), \\
\left\{U(u), \alpha^{-}(v)\right\} & =-\alpha^{-}(u) \delta^{\prime}(u-v), \\
\left\{\alpha^{+}(u), \alpha^{-}(v)\right\} & =-2 U(u) \delta(u-v)-2 V(u) \delta^{\prime}(u-v)-2 \delta^{\prime \prime}(u-v), \\
\left\{V(u), \alpha^{+}(v)\right\} & =-\alpha^{+}(u) \delta(u-v), \quad\left\{V(u), \alpha^{-}(v)\right\}=\alpha^{-}(u) \delta(u-v), \\
\{U(u), V(v)\} & =-V(u) \delta^{\prime}(u-v), \quad\{V(u), V(v)\}=-2 \delta^{\prime}(u-v), \\
\{U(u), U(v)\} & =-U^{\prime}(u) \delta(u-v)-2 U(u) \delta^{\prime}(u-v) .
\end{aligned}
$$

Теперь перепишем матричный $L$-оператор в алгебраической форме

$$
\begin{aligned}
\mathcal{L}_{F}=D & -\left(h_{\alpha_{1}} D \Phi_{1}+h_{\alpha_{2}} D \Phi_{2}+e_{\alpha_{1}}+e_{\alpha_{2}}+\left[e_{\alpha_{2}}, e_{\alpha_{0}}\right]+\right. \\
& \left.+\left[e_{\alpha_{0}}, e_{\alpha_{1}}\right]+D\left(\Phi_{1}-\Phi_{2}\right) e_{\alpha_{0}}\right),
\end{aligned}
$$

где $h_{\alpha_{i}}, e_{\alpha_{i}}$ - генераторы верхней подалгебры Бореля $s l^{(1)}(2 \mid 1)$ со следующими коммутационными соотношениями:

$$
\begin{aligned}
{\left[e_{\alpha_{i}}, e_{-\alpha_{j}}\right] } & =\delta_{i, j} h_{\alpha_{i}}, \quad i, j=0,1,2 ; \\
{\left[h_{\alpha_{j}}, e_{ \pm \alpha_{i}}\right] } & = \pm e_{ \pm \alpha_{i}}, \quad i, j=1,2, \quad i \neq j ; \\
{\left[h_{\alpha_{i}}, e_{ \pm \alpha_{0}}\right] } & =\mp e_{ \pm \alpha_{0}}, \quad\left[h_{\alpha_{0}}, e_{ \pm \alpha_{i}}\right]=\mp e_{ \pm \alpha_{i}}, \quad i=1,2 \\
{\left[h_{\alpha_{0}}, e_{ \pm \alpha_{0}}\right] } & = \pm 2 e_{ \pm \alpha_{0}} ; \quad\left[h_{\alpha_{i}}, e_{ \pm \alpha_{i}}\right]=0, \quad i=1,2 ; \\
\operatorname{ad}_{e_{ \pm \alpha_{i}}}^{2} e_{ \pm \alpha_{j}} & \equiv\left[e_{ \pm \alpha_{i}},\left[e_{ \pm \alpha_{i}}, e_{ \pm \alpha_{j}}\right]\right]=0, \quad i, j=0,1,2 ; \\
{\left[e_{ \pm \alpha_{k}}, e_{ \pm \alpha_{k}}\right] } & =0, \quad k=1,2 .
\end{aligned}
$$

Здесь $e_{ \pm \alpha_{i}}-$ нечетные генераторы для $i=1,2$ и четные генераторы для $i=0$, а $[\cdot, \cdot]$ обозначает суперкоммутатор.

Симметризованная матрица Картана с элементами $b_{i j}=\left(\alpha_{i}, \alpha_{j}\right)$, соответствующая данной алгебре, имеет следующий вид:

$$
b=\left(\begin{array}{ccc}
0 & 1 & -1 \\
1 & 0 & -1 \\
-1 & -1 & 2
\end{array}\right)
$$

6 Теоретическая и математическая физика, т. 147, № 2, 2006 г. 
В этой работе мы будем использовать только так называемые "evaluation"-представления, т.е. представления, в которых центральный заряд соответствующей аффинной алгебры равен нулю.

Для того чтобы записать матрицу монодромии, мы должны рассмотреть эквивалентный бозонный $L$-оператор. Представляя линейную задачу, связанную с оператором (4), следующим образом:

$$
\mathcal{L} \Psi=\left(\partial_{\theta}+\theta \partial_{u}+N_{1}+\theta N_{0}\right)\left(\Psi_{0}+\theta \Psi_{1}\right),
$$

легко переписать ее как бозонную линейную задачу на $\Psi_{0}$ :

$$
\mathcal{L}_{B} \Psi_{0} \equiv\left(\partial_{u}+N_{1}^{2}+N_{0}\right) \Psi_{0}=0
$$

при этом $\Psi_{1}=-N_{1} \Psi_{0}$, где

$$
\begin{gathered}
N_{1}=\frac{i}{\sqrt{2}} \xi_{1} h_{\alpha_{1}}+\frac{i}{\sqrt{2}} \xi_{2} h_{\alpha_{2}}-e_{\alpha_{1}}-e_{\alpha_{2}}-\left[e_{\alpha_{2}}, e_{\alpha_{0}}\right]-\left[e_{\alpha_{0}}, e_{\alpha_{1}}\right]+\frac{i}{\sqrt{2}}\left(\xi_{1}-\xi_{2}\right) e_{\alpha_{0}}, \\
N_{0}=-\phi_{1}^{\prime} h_{\alpha_{1}}-\phi_{2}^{\prime} h_{\alpha_{2}}-\left(\phi_{1}^{\prime}-\phi_{2}^{\prime}\right) e_{\alpha_{0}} .
\end{gathered}
$$

Таким образом,

$$
\begin{aligned}
\mathcal{L}_{B}=\partial_{u} & +\left(\frac{i}{\sqrt{2}} \xi_{1} h_{\alpha_{1}}+\frac{i}{\sqrt{2}} \xi_{2} h_{\alpha_{2}}-\left[e_{\alpha_{2}}, e_{\alpha_{0}}\right]-\left[e_{\alpha_{0}}, e_{\alpha_{1}}\right]-\right. \\
& \left.-e_{\alpha_{1}}-e_{\alpha_{2}}+\frac{i}{\sqrt{2}}\left(\xi_{1}-\xi_{2}\right) e_{\alpha_{0}}\right)^{2}-\left(\phi_{1}^{\prime}-\phi_{2}^{\prime}\right) e_{\alpha_{0}}-\phi_{1}^{\prime} h_{\alpha_{1}}-\phi_{2}^{\prime} h_{\alpha_{2}}
\end{aligned}
$$

Рассматривая соответствующую линейную задачу, можно записать решение в таком виде:

$$
\chi(u)=e^{\sum_{i=1,2} \phi_{i}(u) h_{\alpha_{i}}} \operatorname{Pexp} \int_{0}^{u} d u^{\prime}\left(\sum_{k=0,1,2} W_{\alpha_{k}}\left(u^{\prime}\right) e_{\alpha_{k}}+K\left(u^{\prime}\right)\right),
$$

где

$$
\begin{aligned}
W_{\alpha_{j}}= & \int d \theta e^{-\Phi_{j}}, \quad j=1,2 ; \quad W_{\alpha_{0}}=\int d \theta\left(D \Phi_{1}-D \Phi_{2}\right) e^{\Phi_{1}+\Phi_{2}} \\
K(u)=- & \frac{i}{\sqrt{2}} \xi_{2}\left[e_{\alpha_{2}}, e_{\alpha_{0}}\right] e^{\phi_{2}}-\frac{i}{\sqrt{2}} \xi_{1}\left[e_{\alpha_{0}}, e_{\alpha_{1}}\right] e^{\phi_{1}}-\left[e_{\alpha_{1}}, e_{\alpha_{2}}\right] e^{-\phi_{1}-\phi_{2}}- \\
& \quad-\left[\left[e_{\alpha_{0}}, e_{\alpha_{1}}\right],\left[e_{\alpha_{2}}, e_{\alpha_{0}}\right]\right] e^{\phi_{1}+\phi_{2}}-\left[e_{\alpha_{2}},\left[e_{\alpha_{0}}, e_{\alpha_{1}}\right]\right]-\left[e_{\alpha_{1}},\left[e_{\alpha_{2}}, e_{\alpha_{0}}\right]\right] .
\end{aligned}
$$

Мы можем определить матрицу монодромии на интервале $[0,2 \pi]$ с квазипериодическими граничными условиями

$$
\phi_{j}(u+2 \pi)=\phi_{j}(u)+2 \pi i p_{j}, \quad \xi_{j}(u+2 \pi)= \pm \xi_{j}(u)
$$

следующим образом:

$$
\mathbf{M}=e^{\sum_{i=1,2} 2 \pi p_{i} h_{\alpha_{i}}} \operatorname{Pexp} \int_{0}^{2 \pi} d u\left(\sum_{k=0,1,2} W_{\alpha_{k}}(u) e_{\alpha_{k}}+K(u)\right) .
$$


Причина разделения подынтегрального выражения на $K$-член и ковариантную часть, выраженную через суперполя, состоит в том, что в квантовом случае (см. ниже) нековариантный $K$-член пропадает из выражения для квантовой матрицы монодромии.

Как и в случае стандартных моделей КдФ, определим вспомогательные L-операторы:

$$
\mathbf{L}=e^{-\sum_{i=1,2} i \pi p_{i} h_{\alpha_{i}}} \mathbf{M}
$$

которые удовлетворяют квадратичному r-матричному соотношению [7]

$$
\{\mathbf{L}(\lambda), \otimes \mathbf{L}(\mu)\}=\left[\mathbf{r}\left(\lambda \mu^{-1}\right), \mathbf{L}(\lambda) \otimes \mathbf{L}(\mu)\right]
$$

с восстановленной зависимостью от спектральных параметров $\lambda, \mu$, соответствующих каким-то заданным "evaluation"-представлениям. Здесь $\mathbf{r}\left(\lambda \mu^{-1}\right)$ - это классическая тригонометрическая r-матрица, ассоциированная с $s l^{(1)}(2 \mid 1)$ [8]. Как обычно, это дает соотношение, приводящее к классической интегрируемости:

$$
\{\mathbf{t}(\lambda), \mathbf{t}(\mu)\}=0
$$

где $\mathbf{t}(\lambda)=\operatorname{str} \mathbf{M}(\lambda)$, и суперслед взят в одном из представлений $s l^{(1)}(2 \mid 1)$.

\section{3. КВАНТОВАЯ R-МАТРИЦА И БАЗИС КАРТАНА-ВЕЙЛЯ ДЛЯ $S L_{Q}^{(1)}(2 \mid 1)$}

Квантовая алгебра $s l_{q}^{(1)}(2 \mid 1)$ имеет следующие коммутационные соотношения:

$$
\begin{aligned}
{\left[e_{\alpha_{i}}, e_{-\alpha_{j}}\right] } & =\delta_{i, j}\left[h_{\alpha_{i}}\right], \quad i, j=0,1,2 ; \\
{\left[h_{\alpha_{j}}, e_{ \pm \alpha_{i}}\right] } & = \pm e_{ \pm \alpha_{i}}, \quad i, j=1,2, \quad i \neq j ; \\
{\left[h_{\alpha_{i}}, e_{ \pm \alpha_{0}}\right] } & =\mp e_{ \pm \alpha_{0}}, \quad\left[h_{\alpha_{0}}, e_{ \pm \alpha_{i}}\right]=\mp e_{ \pm \alpha_{i}} . \quad i=1,2 \\
{\left[h_{\alpha_{i}}, e_{ \pm \alpha_{i}}\right] } & =0, \quad i=1,2 ; \\
{\left[h_{\alpha_{0}}, e_{ \pm \alpha_{0}}\right] } & = \pm 2 e_{ \pm \alpha_{0}} ; \quad\left[e_{ \pm \alpha_{i}},\left[e_{ \pm \alpha_{i}}, e_{ \pm \alpha_{j}}\right]_{q}\right]_{q}=0, \quad i, j=0,1,2 \\
e_{\alpha_{k}}^{2} & =0, \quad k=1,2 .
\end{aligned}
$$

Здесь $[x]=\left(q^{x}-q^{-x}\right) /\left(q-q^{-1}\right)$, а квантовый суперкоммутатор определен следующим образом:

$$
\left[e_{\alpha}, e_{\beta}\right]_{q}=e_{\alpha} e_{\beta}-(-1)^{p(\alpha) p(\beta)} q^{(\alpha, \beta)} e_{\beta} e_{\alpha}
$$

Универсальная квантовая $R$-матрица для квантовых аффинных супералгебр имеет вид [9]

$$
R=K \bar{R}=K\left(\prod_{\alpha \in \Delta_{+}} R_{\alpha}\right)
$$

где $\bar{R}$ - редуцированная $R$-матрица, и $R_{\alpha}$ заданы следующими формулами:

$$
R_{\alpha}=\exp _{q_{\alpha}^{-1}}\left\{(-1)^{p(\alpha)}\left(q-q^{-1}\right)(a(\alpha))^{-1}\left(e_{\alpha} \otimes e_{-\alpha}\right)\right\}
$$


для вещественных корней и

$$
R_{n \delta}=\exp \left\{\left(q-q^{-1}\right) \sum_{i, j}^{\text {mult }} c_{i j}(n) e_{n \delta}^{(i)} \otimes e_{-n \delta}^{(j)}\right\}
$$

для чисто мнимых корней. Здесь $\Delta_{+}$- это редуцированная положительная система корней (мы исключаем бозонные корни, являющиеся удвоенными фермионными), а q-экспонента определяется, как обычно:

$$
\exp _{q}(x)=\sum_{n=0}^{\infty} \frac{x^{n}}{(n)_{q} !}, \quad(n)_{q}=\frac{q^{n}-1}{q-1} .
$$

Генераторы, соответствующие составным корням, а также упорядочение в (18), задаются в соответствии с конструкцией базиса Картана-Вейля [9]. Картановский фактор в случае $s l_{q}^{(1)}(1 \mid 2)$ равен $K=q^{h_{\alpha_{1}} \otimes h_{\alpha_{2}}+h_{\alpha_{2}} \otimes h_{\alpha_{1}}}$. Коэффициенты $a(\alpha), c_{i j}(n)$, $d_{i j}(n)$ определяется при помощи следующих соотношений:

$$
\begin{aligned}
{\left[e_{\gamma}, e_{-\gamma}\right] } & =a(\gamma) \frac{k_{\gamma}-k_{\gamma}^{-1}}{q-q^{-1}}, \\
{\left[e_{n \delta}^{(i)}, e_{-n \delta}^{(j)}\right] } & =d_{i j}(n) \frac{q^{n h_{\delta}}-q^{-n h_{\delta}}}{q-q^{-1}},
\end{aligned}
$$

a $c_{i j}(n)$ является обратной матрицей к $d_{i j}(n)$. Первые несколько генераторов, соответствующие составным корням и построенные согласно указанной процедуре, имеют вид

$$
\begin{array}{cc}
e_{\alpha_{1}+\alpha_{2}}=\left[e_{\alpha_{1}}, e_{\alpha_{2}}\right]_{q^{-1}}, \\
e_{\delta-\alpha_{1}} \equiv e_{\alpha_{0}+\alpha_{2}}=\left[e_{\alpha_{0}}, e_{\alpha_{2}}\right]_{q^{-1}}, & e_{\delta-\alpha_{2}} \equiv e_{\alpha_{0}+\alpha_{1}}=\left[e_{\alpha_{1}}, e_{\alpha_{0}}\right]_{q^{-1}}, \\
e_{\delta}^{(1)}=\left[\left[e_{\alpha_{0}}, e_{\alpha_{2}}\right]_{q^{-1}}, e_{\alpha_{1}}\right], & e_{\delta}^{(2)}=\left[\left[e_{\alpha_{1}}, e_{\alpha_{0}}\right]_{q^{-1}}, e_{\alpha_{2}}\right], \\
e_{2 \delta-\alpha_{1}-\alpha_{2}}=\left[e_{\delta-\alpha_{2}}, e_{\delta-\alpha_{1}}\right]_{q^{-1}}, & e_{-\alpha_{1}-\alpha_{2}}=\left[e_{-\alpha_{2}}, e_{-\alpha_{1}}\right]_{q}, \\
e_{-\delta+\alpha_{1}} \equiv e_{-\alpha_{0}-\alpha_{2}}=\left[e_{-\alpha_{2}}, e_{-\alpha_{0}}\right]_{q}, & e_{-\delta+\alpha_{2}} \equiv e_{-\alpha_{0}-\alpha_{1}}=\left[e_{-\alpha_{0}}, e_{-\alpha_{1}}\right]_{q}, \\
e_{-\delta}^{(1)}=\left[\left[e_{-\alpha_{2}}, e_{-\alpha_{0}}\right]_{q}, e_{-\alpha_{1}}\right], & e_{-\delta}^{(2)}=\left[\left[e_{-\alpha_{0}}, e_{-\alpha_{1}}\right]_{q}, e_{-\alpha_{2}}\right], \\
e_{-2 \delta+\alpha_{1}+\alpha_{2}}=\left[e_{-\delta+\alpha_{1}}, e_{-\delta+\alpha_{2}}\right]_{q} .
\end{array}
$$

\section{4. ПОСТРОЕНИЕ КВАНТОВОЙ МАТРИЦЫ МОНОДРОМИИ}

В этом разделе построен квантовый аналог матрицы монодромии, введенный в разделе 2. Показано, что в классическом пределе получается матрица (13).

Вначале рассмотрим квантовые версии экспонент свободных полей (вершинных операторов):

$$
\begin{aligned}
& W_{\alpha_{j}}=\int d \theta: e^{-\Phi_{j}}: \equiv \frac{i}{\sqrt{2}} \xi_{i}: e^{-\phi_{j}}:, \quad j=1,2 \\
& W_{\alpha_{0}}=\int d \theta:\left(D \Phi_{1}-D \Phi_{2}\right) e^{\Phi_{1}+\Phi_{2}}: \equiv: e^{\phi_{1}+\phi_{2}}\left(\phi_{1}^{\prime}-\phi_{2}^{\prime}+\xi_{1} \xi_{2}\right): .
\end{aligned}
$$


Представим суперполя следующим образом: $\Phi_{1}=\left(i \phi_{+}+\phi_{-}\right) / \sqrt{2}, \quad \Phi_{2}=\left(i \phi_{+}-\right.$ $\left.\phi_{-}\right) / \sqrt{2}$, где

$$
\begin{gathered}
\phi_{ \pm}(u)=i Q^{ \pm}+i P^{ \pm} u+\sum_{n} \frac{a_{-n}^{ \pm}}{n} e^{i n u}, \quad \xi_{ \pm}(u)=i^{-1 / 2} \sum_{n} \xi_{n}^{ \pm} e^{-i n u}, \\
{\left[Q^{ \pm}, P^{ \pm}\right]=\frac{i \beta^{2}}{2}, \quad\left[a_{n}^{ \pm}, a_{m}^{ \pm}\right]=\frac{\beta^{2}}{2} n \delta_{n+m, 0}, \quad\left\{\xi_{n}^{ \pm}, \xi_{m}^{ \pm}\right\}=\beta^{2} \delta_{n+m, 0},}
\end{gathered}
$$

а нормальное упорядочение в (22) определяется так:

$$
: e^{c \phi_{ \pm}(u)}:=\exp \left(c \sum_{n=1}^{\infty} \frac{a_{-n}^{ \pm}}{n} e^{i n u}\right) \exp \left(c i\left(Q^{ \pm}+P^{ \pm} u\right)\right) \exp \left(-c \sum_{n=1}^{\infty} \frac{a_{n}^{ \pm}}{n} e^{-i n u}\right) .
$$

Здесь операторы $a_{n}^{ \pm}$с отрицательным $n$ передвинуты налево, а с положительным $n-$ направо. Вершинные операторы (22), проинтегрированные от $u_{1}$ до $u_{2}$, удовлетворяют квантовым соотношениям Серра и так называемым "нестандартным" соотношениям Серра для нижней подалгебры Бореля, где $q=e^{i \pi \beta^{2} / 2}$.

Доказательство данного факта нетривиально, т.к. обычное доказательство соотношений Серра, приведенное в работах [10] для бозонного случая, основывающееся на сведении произведений проинтегрированных вертекс-операторов к упорядоченным интегралам не проходит из-за сингулярностей, образованных фермионными полями в соответствующих операторных произведениях. Однако существует другой метод доказательства, использующий обычную в конформной теории поля технику контурного интегрирования и аналитического продолжения операторных произведений нелокальных вертекс-операторов [11]. Это доказательство годится также для случая квантовой аффинной супералгебры и соответствующих вертекс-операторов, поскольку данный метод позволяет выделить расходимости в каждом из произведений вертекс-операторов и затем их сократить в "стандартных" и "нестандартных" соотношениях Серра. Этот вопрос для произвольной квантовой аффинной супералгебры будет рассмотрен в отдельной работе.

Следствием того, что операторы $\left(q-q^{-1}\right)^{-1} \int_{u_{1}}^{u_{2}} d u W_{\alpha_{i}}$ удовлетворяют соотношениям Серра, является возможность представления нижней борелевской алгебры с помощью соответствия

$$
e_{-\alpha_{i}} \rightarrow\left(q-q^{-1}\right)^{-1} \int_{u_{1}}^{u_{2}} d u W_{\alpha_{i}}
$$

В работе [12] было показано, что соответствующим образом представленная редуцированная $R$-матрица $\bar{R}$, которую мы обозначим $\overline{\mathbf{L}}^{(q)}\left(u_{2}, u_{1}\right)$, обладает свойством Р-экспоненты, удовлетворяя следующему функциональному соотношению:

$$
\overline{\mathbf{L}}^{(q)}\left(u_{3}, u_{1}\right)=\overline{\mathbf{L}}^{(q)}\left(u_{3}, u_{2}\right) \overline{\mathbf{L}}^{(q)}\left(u_{2}, u_{1}\right) .
$$

Однако в суперсимметричном случае, когда появляются фермионные поля, связанные с ними сингулярности в операторных произведениях не позволяют выразить 
$\overline{\mathbf{L}}^{(q)}\left(u_{2}, u_{1}\right)$ стандартным образом, т.е. в терминах упорядоченных интегралов. Поэтому мы назвали ее "квантовой” Р-экспонентой, и в нашем случае она может быть записана как

$$
\overline{\mathbf{L}}^{(q)}\left(u_{2}, u_{1}\right)=\operatorname{Pexp}^{(q)} \int_{u_{1}}^{u_{2}} d u \sum_{k=0,1,2} W_{\alpha_{k}}(u) e_{\alpha_{k}}
$$

Можно показать, что операторы $e^{\sum_{i=1,2} \pi i p_{i} h_{\alpha_{i}}} \overline{\mathbf{L}}^{(q)}(2 \pi, 0)=\mathbf{L}^{(q)}$ удовлетворяют $R T T$ соотношению [13]

$$
\mathbf{R}\left(\lambda \mu^{-1}\right)\left(\mathbf{L}^{(q)}(\lambda) \otimes \mathbf{I}\right)\left(\mathbf{I} \otimes \mathbf{L}^{(q)}(\mu)\right)=\left(\mathbf{I} \otimes \mathbf{L}^{(q)}(\mu)\right)\left(\mathbf{L}^{(q)}(\lambda) \otimes \mathbf{I}\right) \mathbf{R}\left(\lambda \mu^{-1}\right),
$$

где зависимость от $\lambda, \mu$ означает, что мы рассматриваем операторы $\mathbf{L}^{(q)}$ в соответствующих "evaluation"-представлениях $s l_{q}^{(1)}(2 \mid 1)$. Отметим также, что

$$
\mathbf{M}^{(q)}=e^{\sum_{i=1,2} 2 \pi i p_{i} h_{\alpha_{i}}} \overline{\mathbf{L}}^{(q)}
$$

удовлетворяют уравнению отражения [14]

$$
\widetilde{\mathbf{R}}_{12}\left(\lambda \mu^{-1}\right) \mathbf{M}_{1}^{(q)}(\lambda) F_{12}^{-1} \mathbf{M}_{2}^{(q)}(\mu)=\mathbf{M}_{2}^{(q)}(\mu) F_{12}^{-1} \mathbf{M}_{1}^{(q)}(\lambda) \mathbf{R}_{12}\left(\lambda \mu^{-1}\right),
$$

где $F=K^{-1}$ есть обратный картановский фактор из универсальной $R$-матрицы,

$$
\widetilde{\mathbf{R}}_{12}\left(\lambda \mu^{-1}\right)=F_{12}^{-1} \mathbf{R}_{12}\left(\lambda \mu^{-1}\right) F_{12}
$$

а индексы 1, 2 обозначают расположение множителей в тензорном произведении. Это приводит к квантовому соотношению интегрируемости:

$$
\left[\mathbf{t}^{(q)}(\lambda), \mathbf{t}^{(q)}(\mu)\right]=0
$$

где $\mathbf{t}^{(q)}(\lambda)=\operatorname{str} \mathbf{M}^{(q)}(\lambda)$.

Покажем, что $\mathbf{L}^{(q)}, \mathbf{M}^{(q)}$ в классическом пределе $q \rightarrow 1$ переходят во вспомогательную $\mathbf{L}$-матрицу и матрицу монодромии, соответственно. Отметим также, что как обычно, квантовая универсальная $R$-матрица при $q \rightarrow 1$ стремится к классической $r$-матрице, следовательно, классический предел оператора $\mathbf{L}^{(q)}$ реализует классическую $r$-матрицу с помощью классических аналогов соответствующих вертексоператоров.

Для нахождения классического предела разложим $\overline{\mathbf{L}}^{(q)}$ следующим образом [2]:

$$
\overline{\mathbf{L}}^{(q)}(2 \pi, 0)=\lim _{N \rightarrow \infty} \prod_{m=1}^{N} \overline{\mathbf{L}}^{(q)}\left(x_{m}, x_{m-1}\right),
$$

где мы разделили интервал $[0,2 \pi]$ на инфинитезимальные интервалы $\left[x_{m}, x_{m+1}\right]$, $x_{m+1}-x_{m}=\epsilon=2 \pi / N$. Теперь найдем члены, дающие вклад первого порядка по 
$\epsilon$ в $\overline{\mathbf{L}}^{(q)}\left(x_{m}, x_{m-1}\right)$. Для этого нам будут необходимы операторные разложения вершинных операторов. Нетривиальные члены возникают в следующих разложениях:

$$
\begin{aligned}
\xi_{1}(u) \xi_{2}\left(u^{\prime}\right) & =\frac{i \beta^{2}}{\left(i u-i u^{\prime}\right)}+\sum_{k=0}^{\infty} c_{k}(u)\left(i u-i u^{\prime}\right)^{k} \\
: e^{a \phi_{1}(u)}:: e^{b \phi_{2}\left(u^{\prime}\right)}: & =\left(i u-i u^{\prime}\right)^{\frac{-a b \beta^{2}}{2}}\left(: e^{\left(a \phi_{1}(u)+b \phi_{2}(u)\right.}:+\sum_{k=1}^{\infty} d_{k}(u)\left(i u-i u^{\prime}\right)^{k}\right), \\
\phi_{1}^{\prime}(u): e^{b \phi_{2}\left(u^{\prime}\right)}: & =\frac{-i b \beta^{2}: e^{b \phi_{2}(u)}:}{2\left(i u-i u^{\prime}\right)}+\sum_{k=0}^{\infty} f_{k}(u)\left(i u-i u^{\prime}\right)^{k} \\
\phi_{2}^{\prime}(u): e^{b \phi_{1}\left(u^{\prime}\right)}: & =\frac{-i b \beta^{2}: e^{b \phi_{1}(u)}:}{2\left(i u-i u^{\prime}\right)}+\sum_{k=0}^{\infty} f_{k}(u)\left(i u-i u^{\prime}\right)^{k} .
\end{aligned}
$$

В случаях, рассмотренных в работе [2], только два типа членов давали вклад порядка $\epsilon$ в $\overline{\mathbf{L}}^{(q)}\left(x_{m-1}, x_{m}\right)$ при $q \rightarrow 1$. $\mathrm{K}$ членам первого типа относятся операторы первого порядка по $W_{\alpha_{i}}$, а второй тип образован операторами, квадратичными по $W_{\alpha_{i}}$, которые дают вклад порядка $\epsilon^{1 \pm \beta^{2}}$ в силу своего операторного разложения. Вклады второго типа соответствуют составным корням, являющимся суммой двух простых.

В данной работе мы покажем, что имеются вклады составных корней, равные сумме трех и даже четырех корней, которые обеспечивают необходимые члены в классических выражениях (12), (13). Сначала рассмотрим квадратичные члены, соответствующие отрицательным корням $-\alpha_{1}-\alpha_{2},-\delta+\alpha_{2},-\delta+\alpha_{1}$. Коммутационные соотношения между вершинными операторами на окружности устроены таким образом, что при $u>u^{\prime}$

$$
\begin{aligned}
& W_{\alpha_{i}}(u) W_{\alpha_{j}}\left(u^{\prime}\right)=-q^{-1} W_{\alpha_{j}}\left(u^{\prime}\right) W_{\alpha_{i}}(u), \quad i, j=1,2 \quad i \neq j ; \\
& W_{\alpha_{i}}(u) W_{\alpha_{0}}\left(u^{\prime}\right)=q W_{\alpha_{0}}\left(u^{\prime}\right) W_{\alpha_{i}}(u) ; \\
& W_{\alpha_{0}}(u) W_{\alpha_{i}}\left(u^{\prime}\right)=q W_{\alpha_{i}}\left(u^{\prime}\right) W_{\alpha_{0}}(u), \quad i=1,2 .
\end{aligned}
$$

Это позволяет нам записать генераторы, соответствующие отрицательным составным корням $-\delta+\alpha_{2},-\delta+\alpha_{1}$, в следующем виде:

$$
\begin{aligned}
{\left[e_{-\alpha_{0}}, e_{-\alpha_{1}}\right]_{q} } & =\frac{1}{q-q^{-1}} \int_{x_{m-1}}^{x_{m}} d u W_{\alpha_{1}}(u) \int_{x_{m-1}}^{u} d u^{\prime} W_{\alpha_{0}}\left(u^{\prime}\right), \\
{\left[e_{-\alpha_{2}}, e_{-\alpha_{0}}\right]_{q} } & =\frac{1}{q-q^{-1}} \int_{x_{m-1}}^{x_{m}} d u W_{\alpha_{0}}(u) \int_{x_{m-1}}^{u} d u^{\prime} W_{\alpha_{2}}\left(u^{\prime}\right) .
\end{aligned}
$$

Показатели соответствующих $q$-экспонент в квантовой $R$-матрице (18) равны

$$
\begin{aligned}
& \int_{x_{m-1}}^{x_{m}} d u W_{\alpha_{1}}(u) \int_{x_{m-1}}^{u} d u^{\prime} W_{\alpha_{0}}\left(u^{\prime}\right)\left[e_{\alpha_{1}}, e_{\alpha_{0}}\right]_{q^{-1}} \\
& \int_{x_{m-1}}^{x_{m}} d u W_{\alpha_{0}}(u) \int_{x_{m-1}}^{u} d u^{\prime} W_{\alpha_{2}}\left(u^{\prime}\right)\left[e_{\alpha_{0}}, e_{\alpha_{2}}\right]_{q^{-1}} .
\end{aligned}
$$


В классическом пределе $\left(\beta^{2} \rightarrow 0\right)$ их вклад, вычисленный с помощью соответствующих операторных разложений (аналогично работе [1]), имеет вид

$$
\int_{x_{m-1}}^{x_{m}} d u\left(-\frac{i}{\sqrt{2}} \xi_{2} e^{\phi_{2}}\left[e_{\alpha_{2}}, e_{\alpha_{0}}\right]-\frac{i}{\sqrt{2}} \xi_{1} e^{\phi_{1}}\left[e_{\alpha_{0}}, e_{\alpha_{1}}\right]\right) .
$$

Вклад корня $-\alpha_{1}-\alpha_{2}$ равен

$$
\int_{x_{m-1}}^{x_{m}} d u\left(-e^{-\phi_{1}-\phi_{2}}\left[e_{\alpha_{1}}, e_{\alpha_{2}}\right]\right) .
$$

В этом случае коммутатор проинтегрированных вершинных операторов не может быть переписан в терминах упорядоченных интегралов, как это было проделано выше. Используем тот факт, что проинтегрированные вершинные операторы должны быть радиально упорядочены, т.е., например, произведение $e_{-\alpha_{k}} e_{-\alpha_{j}}$ должно быть записано как

$$
\int_{x_{m-1}}^{x_{m}} d u W_{\alpha_{k}}(u-i 0) \int_{x_{m-1}}^{x_{m}} d u^{\prime} W_{\alpha_{j}}\left(u^{\prime}+i 0\right),
$$

а также учтем известное соотношение

$$
\frac{1}{x+i 0}-\frac{1}{x-i 0}=-2 i \pi \delta(x)
$$

В результате, проведя вычисления, обобщающие результаты работы [2], мы получим формулу (35). Таким образом, на “квадратичном" уровне имеется полное согласие с классическим выражением.

Теперь рассмотрим вклад, соответствующий составным корням $e_{\delta}^{(i)}$ и $2 \delta-\alpha_{1}-\alpha_{2}$. Сначала мы рассмотрим чисто мнимые корни

$$
e_{-\delta}^{(1)}=\left[\left[e_{-\alpha_{2}}, e_{-\alpha_{0}}\right]_{q}, e_{-\alpha_{1}}\right], \quad e_{-\delta}^{(2)}=\left[\left[e_{-\alpha_{0}}, e_{-\alpha_{1}}\right]_{q}, e_{-\alpha_{2}}\right] .
$$

Чтобы вычислить их вклад в классическом пределе, мы должны учесть члены порядка $\epsilon^{1+\beta^{2}}$, пропорциональные $\int d u d \theta e^{\Phi_{2}}$ и $\int d u d \theta e^{\Phi_{1}}$, в $\left[e_{\alpha_{2}}, e_{\alpha_{0}}\right]_{q}$ и $\left[e_{\alpha_{0}}, e_{\alpha_{1}}\right]_{q}$, соответственно. Затем мы рассмотрим их суперкоммутаторы с $e_{\alpha_{1}}$ и $e_{\alpha_{2}}$. Переписывая эти суперкоммутаторы в терминах упорядоченных интегралов, как это было выше, мы находим, следуя вычислениям из [1], что вклад соответствующего показателя $q$-экспоненты имеет следующий вид:

$$
\int_{x_{m-1}}^{x_{m}} d u\left(-\left[e_{\alpha_{2}},\left[e_{\alpha_{0}}, e_{\alpha_{1}}\right]\right]-\left[e_{\alpha_{1}},\left[e_{\alpha_{2}}, e_{\alpha_{0}}\right]\right]\right) .
$$

Генератор $e_{2 \delta-\alpha_{1}-\alpha_{2}}$ выражается аналогично в терминах $q$-коммутатора $\left[e_{\alpha_{2}}, e_{\alpha_{0}}\right]_{q}$ и $\left[e_{\alpha_{0}}, e_{\alpha_{1}}\right]_{q}$. Учитывая члены порядка $\epsilon^{1+\beta^{2}}$ и используя формулу $(36)$, как было указано выше, мы получаем, что вклад порядка $\epsilon$ в классическое выражение есть

$$
\int_{x_{m-1}}^{x_{m}} d u\left(-\left[\left[e_{\alpha_{0}}, e_{\alpha_{1}}\right],\left[e_{\alpha_{2}}, e_{\alpha_{0}}\right]\right] e^{\phi_{1}+\phi_{2}}\right) .
$$


Следовательно, с учетом всех имеющихся членов, первая итерация $\overline{\mathbf{L}}$-оператора в классическом пределе имеет вид

$$
\begin{aligned}
\lim _{q \rightarrow 1} & \overline{\mathbf{L}}^{(q)}\left(x_{m}, x_{m-1}\right)=1+\int_{x_{m-1}}^{x_{m}} d u\left(\sum_{k=0,1,2} W_{\alpha_{k}}(u) e_{\alpha_{k}}-\frac{i}{\sqrt{2}} \xi_{2}\left[e_{\alpha_{2}}, e_{\alpha_{0}}\right] e^{\phi_{2}}-\right. \\
& -\frac{i}{\sqrt{2}} \xi_{1}\left[e_{\alpha_{0}}, e_{\alpha_{1}}\right] e^{\phi_{1}}-\left[e_{\alpha_{1}}, e_{\alpha_{2}}\right] e^{-\phi_{1}-\phi_{2}}-\left[\left[e_{\alpha_{0}}, e_{\alpha_{1}}\right],\left[e_{\alpha_{2}}, e_{\alpha_{0}}\right]\right] e^{\phi_{1}+\phi_{2}}- \\
& \left.-\left[e_{\alpha_{2}},\left[e_{\alpha_{0}}, e_{\alpha_{1}}\right]\right]-\left[e_{\alpha_{1}},\left[e_{\alpha_{2}}, e_{\alpha_{0}}\right]\right]\right)+O\left(\epsilon^{2}\right) .
\end{aligned}
$$

Собирая все инфинитезимальные $\overline{\mathbf{L}}$-операторы и умножая на соответствующий картановский фактор, мы получаем искомое классическое выражение для $\mathbf{L}$-операторов и матрицы монодромии.

\section{5. ЗАКЛЮЧЕНИЕ}

Матрица монодромии, предъявленная в предыдущем разделе, построена на основании метода, предложенного в работах [1]-[4], [12]. Другими словами, нами показано, что квантовый аналог вспомогательной L-матрицы совпадает с универсальной $R$-матрицей, в которой нижняя подалгебра Бореля представлена подходящими вершинными операторами. Эта конструкция также позволяет показать, что генераторы суперсимметрии коммутируют с суперследом матрицы монодромии, т.е. они могут быть включены в семейство интегралов движения. Действительно, коммутаторы генераторов суперсимметрии

$$
G_{0}^{+}=\beta^{-2} \sqrt{2} i^{-1 / 2} \int_{0}^{2 \pi} d u \phi_{1}^{\prime}(u) \xi_{2}(u), \quad G_{0}^{-}=\beta^{-2} \sqrt{2} i^{-1 / 2} \int_{0}^{2 \pi} d u \phi_{2}^{\prime}(u) \xi_{1}(u)
$$

с вершинными операторами редуцируются к полным производным; дальнейшие рассуждения полностью повторяют аргументацию работы [3] (см. часть 4), где в случае стандартных иерархий Дринфельда-Соколова было показано (следуя [12]), что оператор суперсимметрии коммутирует со следом матрицы монодромии, если система простых корней является чисто фермионной (при этом условие нечетности системы простых корней гарантирует, что коммутаторы генератора суперсимметрии с соответствующими вертекс-операторами являются полными производными). Аналогично можно показать, что трансфер-матрицы коммутируют с нулевой модой $U(1)$-тока (квантового аналога поля $V$ из (6)), присутствующего в $N=2$ суперконформной алгебре.

Этот результат имеет важные следствия. Если сделать твист-преобразование [15] в $N=2$ суперконформной алгебре, мы получим, что один из генераторов $G_{0}^{ \pm}$становится БРСТ-оператором, т.е. трансфер-матрицы становятся БРСТ-точными, генерируя бесконечное семейство “физических" интегралов движения (с нулевым духовым числом, как показывают коммутационные соотношения с нулевой модой $U(1)$ тока). Это дает нам возможность изучать двумерные топологические модели и их интегрируемые возмущения с помощью методов интегрируемых теорий, например, с помощью хорошо известного квантового метода обратной задачи [13]. 
Благодарности. Автор благодарен рецензенту за ценные замечания и фондам “Династия", CRDF (грант N RUMI-2622-ST-04) и РФФИ (грант N 05-01-00922) за поддержку его работы. Автор особенно признателен Н. Ю. Решетихину, М. А. Семенову-Тян-Шанскому и А. А. Цейтлину за поддержку.

\section{Список литературы}

[1] P. P. Kulish, A. M. Zeitlin, Phys. Lett. B, 581 (2004), 125; hep-th/0312159; П. П. Кулиш, А. М. Цейтлин, ТМФ, 142 (2005), 252; hep-th/0501018.

[2] P. P. Kulish, A. M. Zeitlin, Phys. Lett. B, 597 (2004), 229; hep-th/0407154; Nucl. Phys. B, 709 (2005), 578; hep-th/0501019.

[3] P.P. Kulish, A. M. Zeitlin, Nucl. Phys. B, 720 (2005), 289; hep-th/0506027.

[4] V.V. Bazhanov, S. L. Lukyanov, A. B. Zamolodchikov, Commun. Math. Phys., 177 (1996), 381; 190 (1997), 247; 200 (1999), 297.

[5] F. Delduc, L. Gallot, Supersymmetric Drinfeld-Sokolov reduction, solv-int/9802013.

[6] T. Inami, H. Kanno, Nucl. Phys. B, 359 (1991), 201.

[7] Л. А. Тахтаджян, Л. Д. Фаддеев, Гамильтонов подход в теории солитонов, Наука, M., 1986.

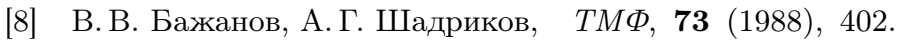

[9] S. M. Khoroshkin, V. N. Tolstoy, Twisting of quantum (super)algebras. Connection of Drinfeld's and Cartan-Weyl realizations for quantum affine algebras, hep-th/9404036.

[10] B. Feigin, E. Frenkel, "Integrals of motion and quantum groups", Integrable Systems and Quantum Groups (Montecatini Terme, Italy, June 14-22, 1993), Lect. Notes Math., 1620, eds. M. Francaviglia, S. Greco, Springer, Berlin, 1996, 349; P. Bouwknegt, J. McCarthy, K. Pilch, hep-th/9110007.

[11] S. Klevtsov, On vertex operator construction of quantum affine algebras, hep-th/0110148.

[12] V. V. Bazhanov, A. N. Hibberd, S. M. Khoroshkin, Nucl. Phys. B, 622 (2002), 475.

[13] L. D. Faddeev, "How the algebraic Bethe ansatz works for integrable models", Quantum symmetries / Symétries quantiques. Proc. of the Les Houches Summer School, Session LXIV (Les Houches, France, August 1 - September 8, 1995), eds. A. Connes, K. Gawedzki, J. Zinn-Justin, Elsevier, Amsterdam, 1998, 149; hep-th/9605187; P.P. Kulish, E. K. Sklyanin, "Quantum spectral transform method: recent development", Integrable Quantum Field Theories. Lect. Notes Phys., 151, eds. J. Hietarinta, C. Montonen, Springer, Berlin, 1982, 61.

[14] P. P. Kulish, E. K. Sklyanin, J. Phys. A, 25 (1992), 5963; P. P. Kulish, R. Sasaki, Prog. Theor. Phys., 89 (1993), 741.

[15] W. Lerche, C. Vafa, N. P. Warner, Nucl. Phys. B, 324 (1989), 427.

Поступила в редакцию 19.IX.2005 г., после доработки 16.XI.2005 г. 